\title{
Modelos populacionais e censo no ensino médio
}

\author{
José Rafael Santos Furlanetto(iD) Ligia Liani Barz묘
}

\begin{abstract}
Resumo
Este trabalho tem como objetivos oferecer uma proposta alternativa de ensino de conteúdos de matemática do ensino médio relacionando-os com temas atuais, e mostrar a importância de manter ou atingir um nível de excelência nos cursos de formação de Licenciados em Matemática. Para isso, como 2020 é um ano bastante importante para o Brasil, pois será realizado o censo demográfico da população brasileira, escolhemos três modelos de crescimento populacional, para fazer um estudo comparativo com os dados oficiais do IBGE. Como os modelos envolvem teoria de equações diferenciais ordinárias do ensino superior, foram discretizados pelo Método de Euler, estabelecendo, dessa forma, uma ligação com os conteúdos do ensino médio.
\end{abstract}

Palavras-chave: Equações Diferenciais Ordinárias; Método de Euler; Modelos Populacionais; Ensino de matemática.

\begin{abstract}
This work aims to offer an alternative proposal for teaching high school mathematics content relating them to current topics and to show the importance of maintaining or achieve a level of excellence in the training courses of Graduates in Mathematics. For this, as 2020 is a very important year for Brazil because the demographic census of the Brazilian population will be carried out, we chose three models of population growth to make a comparative study with the official data from IBGE. As the models involve theory of ordinary differential equations in higher education, they were discretized by the Euler Method, thus establishing a connection with the contents of high school.
\end{abstract}

Keywords: Ordinary Differential Equations, Euler's Method; Population Models; Mathematics Teaching.

\section{Introdução}

Entre agosto e outubro de 2020 ocorrerá mais uma vez a coleta de dados populacionais do Brasil referentes ao censo organizado pelo IBGE. Portanto, julgamos ocasional propor através deste trabalho uma atividade didática na qual o professor possa estimar a população brasileira com seus alunos, bem como gerar a oportunidade para que se discutam itens como a precisão de modelos científicos, como é feita a estimativa oficial dada pelo IBGE, o alcance e o que realmente é ciência, e, claro, proporcionar a chance de que o professor contextualize e aprimore o entendimento dos conceitos matemáticos envolvidos no processo. Para isso, escolhemos três modelos populacionais 
amplamente conhecidos e, de certo modo, um tanto simplificados também, em comparação ao método oficial adotado.

O primeiro modelo tratado foi o Exponencial, seguido do modelo Logístico e, por fim, o modelo de Gompertz, que matematicamente são descritos pelas Equações Diferenciais Ordinárias,

$$
\begin{aligned}
& \text { Exponencial: } \quad \frac{\mathrm{dP}}{\mathrm{dt}}=\mathrm{rP} \\
& \text { Logístico: } \quad \frac{\mathrm{dP}}{\mathrm{dt}}=\mathrm{r}\left(1-\frac{\mathrm{P}}{\mathrm{K}}\right) \mathrm{P} \\
& \text { Gompertz: } \quad \frac{\mathrm{dP}}{\mathrm{dt}}=\mathrm{rP} \ln \left(\frac{\mathrm{K}}{\mathrm{P}}\right)
\end{aligned}
$$

Discretizamos cada uma delas pelo Método de Euler, e utilizamos os dados populacionais brasileiros dados na Tabela 1a para gerar os respectivos fatores r's de cada período que surgem nas expressões desses modelos e estão ligados à taxa de crescimento da população brasileira. Com as tabelas desses fatores de cada modelo, calculamos valores médios para $\mathrm{r}$ através de médias aritmética e harmônica, e adotamos tais valores para estimar a população brasileira de 2020, 2030, 2040, 2050 e 2060, que é até onde vai a estimativa oficial do IBGE conforme a Tabela 1b.

Este é um trabalho voltado ao professor e, portanto, cabe a ele moldar o que considerar necessário a atividade didática que será exposta aqui nas próximas páginas. Daremos sugestões sobre como implementá-la em sala de aula, mas sabemos que apenas o professor conhece a sua turma. Desse modo, estimulamos fortemente que outras formas de aplicar sejam pensadas.

Sendo assim, o trabalho está dividido da seguinte forma. Inicialmente falamos um pouco sobre o Censo que irá ocorrer neste ano de 2020. Trata-se de uma seção apenas ilustrativa, e se houver a necessidade de informações mais profundas, então, o próprio website do IBGE pode ser acessado e deverá bastar. Na segunda parte expomos a matemática utilizada na atividade, desde o método de Euler, passando pelos modelos escolhidos até a compilação dos dados em tabelas e figuras. $\mathrm{Na}$ parte final, além de concluirmos a proposta de atividade didática, discutimos e propomos reflexões sobre temas associados como modelos científicos e seu alcance, a necessidade da formação sólida em matemática avançada para alunos da Licenciatura em Matemática e passos adicionais no aprimoramento da atividade em si.

\subsection{Censo Brasileiro}

Como já citado anteriormente, em 2020 ocorre mais uma contagem da população brasileira. Na realidade tal processo já começou e trata-se de algo de suma importância para o país, uma vez que a precisão desses dados fornece parâmetros sólidos para tomadas de decisões em políticas públicas e demais investimentos tanto privados como por parte dos governos municipais, estaduais e federal.

Como cita o próprio website do IBGE, o recenseamento é constituído de múltiplas etapas, o que deixa à mostra a complexidade do processo, com algumas delas acontecendo desde o ano de 2018. As etapas são:

- Discussão interna.

- Consulta pública.

- Consulta a usuários.

- Consulta à Comissão Consultiva. 
- Testes de questionário.

- Teste de preenchimento pela internet.

- Primeira Prova Piloto.

- Segunda Prova Piloto.

- Censo Experimental.

- Censo Demográfico 2020.

- Divulgação dos Resultados.

É apenas na etapa Censo Demográfico 2020 que a população, de um modo geral tem contato com os recenseadores, etapa essa prevista para ocorrer entre Agosto e Outubro de 2020. As demais são preparatórias e/ou de teste com pequenas parcelas da população. Para saber mais, visite o website do IBGE [7].

As Tabelas 1a e 1b contêm informações de censos demográficos da população brasileira obtidos do website do IBGE, cujos dados foram usados para comparar com os resultados dos modelos propostos neste trabalho. A primeira refere-se aos censos demográficos de 1872 a 2010, e a segunda, trata de estimativas realizadas pelo IBGE no período de 2020 a 2060.

\begin{tabular}{ccc}
\hline $\mathrm{h}$ & Ano & População \\
\hline & 1872 & 9.930 .478 \\
18 & 1890 & 14.333 .915 \\
10 & 1900 & 17.438 .434 \\
20 & 1920 & 30.635 .605 \\
20 & 1940 & 41.236 .315 \\
10 & 1950 & 51.944 .397 \\
10 & 1960 & 70.992 .343 \\
10 & 1970 & 94.508 .583 \\
10 & 1980 & 121.150 .573 \\
11 & 1991 & 146.917 .459 \\
9 & 2000 & 169.590 .693 \\
10 & 2010 & 190.755 .799 \\
\hline
\end{tabular}

\begin{tabular}{ccc}
\hline $\mathrm{h}$ & Ano & População \\
\hline 10 & 2020 & 211.755 .692 \\
10 & 2030 & 224.868 .462 \\
10 & 2040 & 231.919 .922 \\
10 & 2050 & 232.933 .276 \\
10 & 2060 & 228.286 .347 \\
\hline
\end{tabular}

(b)

(a)

Tabela 1: (a) Censo demográfico da população brasileira. (b) Estimativas do IBGE para a população brasileira. Fonte: IBGE

\section{Estimando a população brasileira}

\subsection{O Método de Euler}

Há uma razão bastante relevante para fazermos uma abordagem discreta desses modelos de crescimento populacional: o fato de que temos dados disponíveis de recenseamento em intervalos de alguns anos, como pode ser visto na Tabela 1a. Dessa forma, podemos descrever o tamanho da população por uma sequência $\left\{\mathrm{P}_{\mathrm{n}}\right\}$ com $\mathrm{P}_{0}$ denotando a população inicial, $\mathrm{P}_{1}$ o tamanho da população depois de um tempo $t_{1}$ e assim por diante. 
O método de Euler (ou método da reta tangente) é o mais antigo e mais simples dentre os métodos numéricos que visam aproximar a solução de uma dada Equação Diferencial Ordinária em determinados pontos-chave de interesse no seu domínio de funcionamento.

Resumidamente, ele pode ser descrito a partir da EDO

$$
y^{\prime}=f(t, y)
$$

com uma aproximação da derivada $\mathrm{y}^{\prime}$ por

$$
\frac{y\left(t_{n+1}\right)-y\left(t_{n}\right)}{t_{n+1}-t_{n}}
$$

e subsequente substituição na Equação (1), gerando

$$
\frac{y\left(t_{n+1}\right)-y\left(t_{n}\right)}{t_{n+1}-t_{n}}=f\left(t_{n}, y\left(t_{n}\right)\right),
$$

onde $t_{n}$ e $t_{n+1}$ são pontos determinados no domínio de atuação da equação diferencial. Assim, fazendo $\mathrm{y}\left(\mathrm{t}_{\mathrm{n}}\right)=\mathrm{y}_{\mathrm{n}}$ obtemos

$$
\mathrm{y}_{\mathrm{n}+1}=\mathrm{y}_{\mathrm{n}}+\left(\mathrm{t}_{\mathrm{n}+1}-\mathrm{t}_{\mathrm{n}}\right) \cdot \mathrm{f}\left(\mathrm{t}_{\mathrm{n}}, \mathrm{y}_{\mathrm{n}}\right) \text {. }
$$

Chamando o tamanho do passo no domínio entre os pontos-chave, de $\mathrm{h}_{\mathrm{n}}$, temos

$$
\mathrm{h}_{\mathrm{n}}=\mathrm{t}_{\mathrm{n}+1}-\mathrm{t}_{\mathrm{n}}
$$

e obtemos o formato final usado neste texto:

$$
\mathrm{y}_{\mathrm{n}+1}=\mathrm{y}_{\mathrm{n}}+\mathrm{h}_{\mathrm{n}} \cdot \mathrm{f}\left(\mathrm{t}_{\mathrm{n}}, \mathrm{y}_{\mathrm{n}}\right) \text {. }
$$

Para uma descrição suficientemente adequada desse método, sugerimos o Capítulo 8 da referência $[2]$.

\subsection{Modelos Exponencial e Logístico}

O modelo de crescimento exponencial começa com a hipótese de que

$$
\left\{\begin{array}{l}
\frac{\mathrm{dP}}{\mathrm{dt}}=\mathrm{rP} \\
\mathrm{P}(0)=\mathrm{P}_{0}
\end{array}\right.
$$

onde $\mathrm{P}$ representa o tamanho de uma população no tempo t, $\mathrm{P}_{0}$ é a população inicial, e a constante $\mathrm{r}$ representa a velocidade específica de crescimento, quando $\mathrm{r}>0$, ou decrescimento, quando $\mathrm{r}<0$. Em outras palavras, $\mathrm{r}=\alpha-\beta$ representa a taxa líquida da população definida como a diferença entre as taxas de nascimento $\alpha$ e óbito $\beta$, com $\alpha$ e $\beta$ constantes. O valor de $\mathrm{r}$ é, em geral, calculado para que as taxas de crescimento sejam comparadas em diferentes períodos de tempo (anos, décadas etc.).

A Solução Geral do modelo exponencial é dada por

$$
\mathrm{P}(\mathrm{t})=\mathrm{P}_{0} \mathrm{e}^{\mathrm{rt}}
$$

Neste modelo, a suposição de que a população aumenta a uma taxa proporcional ao número dessa população em cada instante de tempo (3) implica que a população aumenta exponencialmente com 


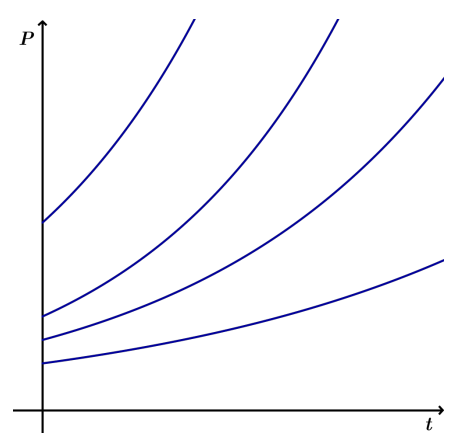

Figura 1: Esboço da Solução Geral da equação exponencial para r > 0 .

o tempo (4), como mostrado na Figura 1. Ou seja, o modelo prevê um crescimento ilimitado da população se $r>0$, a extinção se $r<0$ ou nenhuma alteração se $r=0$.

Uma das primeiras tentativas de se modelar matematicamente o crescimento da população humana foi feita pelo sociólogo e economista inglês Thomas Robert Malthus (1766-1834) e por esta razão o modelo exponencial também é conhecido como modelo de Malthus. Seu primeiro artigo sobre populações, intitulado An Essay on the Principle of Population [9], é de 1798, e foi publicado anonimamente. Em 1807, o artigo [9] é novamente publicado, com Malthus assumindo a autoria.

Esse modelo é simples mas falha ao desconsiderar outros fatores que podem influenciar o crescimento ou o decrescimento da população humana, tais como limitações de espaço e suprimento de alimentos, doenças, imigração e emigração. No entanto, ele foi razoavelmente preciso na previsão da população dos Estados Unidos durante os anos de 1790 a 1890 [10]. Esse modelo funciona bem para modelar o crescimento de populações de bactérias em uma placa de laboratório, e um ótimo exemplo de aplicação em sala de aula é o trabalho [4].

Uma vez que tais limitações ocorrem, pode-se pensar em um modelo que não considera um crescimento exponencial indefinidamente. A versão mais simples é obtida quando a constante $\mathrm{r}$ é substituída por uma função $f(P)$ que dependa do tamanho da população

$$
\frac{\mathrm{dP}}{\mathrm{dt}}=\mathrm{f}(\mathrm{P}) \mathrm{P} .
$$

A escolha de $f(P)$ é feita de modo que $f(P) \simeq r>0$ quando a população for pequena, que decresça com o crescimento de $\mathrm{P}$, e que seja negativa quando a população for suficientemente grande. A forma matemática mais simples com essas características é a função linear

$$
f(P)=r-a P,
$$

sendo a uma constante positiva. Substituindo a Equação (6) na Equação (5) e com alguma manipulação algébrica, obtemos

$$
\left\{\begin{array}{l}
\frac{\mathrm{dP}}{\mathrm{dt}}=\mathrm{r}\left(1-\frac{\mathrm{P}}{\mathrm{K}}\right) \mathrm{P} \\
\mathrm{P}(0)=\mathrm{P}_{0}
\end{array}\right.
$$

onde $\mathrm{K}=\frac{\mathrm{r}}{\mathrm{a}}>0$ é denominada capacidade de carga da população, e representa o tamanho da população que pode ser suportado pelos recursos disponíveis. A EDO em (7) é conhecida como equação Logística ou equação de Verhulst. Foi em 1838 que o matemático belga Pierre-François Verhulst (1804-1849) introduziu a Equação (7) como modelo de crescimento da população humana [11]. 
A Solução Geral do modelo de Verhulst (7) é

$$
\mathrm{P}(\mathrm{t})=\frac{\mathrm{P}_{0}}{\frac{\mathrm{P}_{0}}{\mathrm{~K}}+\left(1-\frac{\mathrm{P}_{0}}{\mathrm{~K}}\right) \mathrm{e}^{-\mathrm{rt}}}
$$

e podemos visualizar o comportamento dessa família de soluções na Figura 2 nos intervalos onde $0<\mathrm{P}_{0}<\frac{\mathrm{K}}{2}, \frac{\mathrm{K}}{2}<\mathrm{P}_{0}<\mathrm{K}$ e $\mathrm{P}_{0}>\mathrm{K}$.

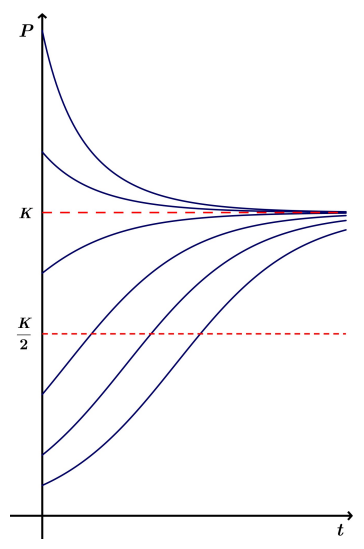

Figura 2: Esboço da Solução Geral da equação logística.

Esse comportamento está de acordo com o comportamento observado de muitas populações. Dessa forma, tal modelo de crescimento populacional com recursos limitados serve de base para muitos modelos em ecologia.

\subsubsection{Discretização da equação exponencial}

Aplicando o modelo de Euler (2) na equação diferencial do modelo Exponencial (3), obtemos

$$
P(n+1)=P(n)\left(1+h_{n} r_{n}\right),
$$

que pode ser escrita na forma

$$
\mathrm{r}_{\mathrm{n}}=\frac{1}{\mathrm{~h}_{\mathrm{n}}}\left(\frac{\mathrm{P}(\mathrm{n}+1)}{\mathrm{P}(\mathrm{n})}-1\right) .
$$

Note que aqui adotamos $\mathrm{r}_{\mathrm{n}}$ em vez de $\mathrm{r}$ pois haverá dependência do passo $\mathrm{n}$ em cada caso. Com a Equação (10) e os valores de h e $\mathrm{P}$ da Tabela 1a, calculamos os valores de $\mathrm{r}_{\mathrm{n}}$ cujos resultados encontram-se na Tabela 2 no final desta subseção. Usando esse modelo, para fazer uma previsão do número de habitantes que o Brasil terá em 2020, devemos encontrar um valor para r que sirva para o período de 2010 a 2020. Como não podemos usar a Equação (10), pois não sabemos qual é a população em 2020 (é exatamente o que queremos determinar), vamos estimar um valor para r usando todos os valores anteriormente calculados e lançando mão, aqui e nos demais modelos tratados, da média aritmética (i) e da média harmônica (ii). Assim,

(i) $\mathrm{r}_{\mathrm{ma}}=\frac{\sum_{\mathrm{i}=1}^{11} \mathrm{r}_{\mathrm{i}}}{11}=\frac{0,2743}{11}=0,0249$; 
(ii) $\mathrm{r}_{\mathrm{mh}}=\frac{11}{\sum_{\mathrm{i}=1}^{11} \frac{1}{\mathrm{r}_{\mathrm{i}}}}=\frac{11}{492,667}=0,0223$.

Usando o valor de $\mathrm{h}_{12}=2020-2010=10$ e o modelo Exponencial discretizado (9),

$$
\mathrm{P}(12)=\mathrm{P}(11)\left(1+\mathrm{h}_{12} \mathrm{r}\right)
$$

obtemos as seguintes projeções da população para 2020:

(i) $\mathrm{P}(12)=238.253 .993$ habitantes com a média aritmética;

(ii) $\mathrm{P}(12)=233.294 .342$ habitantes com a média harmônica.

A Tabela 1b com a estimativa do IBGE [7] para 2020 diz-nos que a população deve ser de 211.755.692 habitantes. Vemos, portanto, que o modelo exponencial está superestimando o crescimento da população brasileira. Mais adiante faremos um paralelo percentual entre todos os modelos em relação às previsões oficiais.

\subsubsection{Discretização da equação logística}

Aplicando o modelo de Euler (2) na equação diferencial do modelo Logístico (7), obtemos

$$
\mathrm{P}(\mathrm{n}+1)=\mathrm{P}(\mathrm{n})+\mathrm{h}_{\mathrm{n}} \mathrm{r}_{\mathrm{n}}\left(1-\frac{\mathrm{P}(\mathrm{n})}{\mathrm{K}}\right) \mathrm{P}(\mathrm{n})
$$

que, com alguma manipulação algébrica, é escrita na forma

$$
\mathrm{r}_{\mathrm{n}}=\frac{\mathrm{K}}{\mathrm{h}_{\mathrm{n}}(\mathrm{K}-\mathrm{P}(\mathrm{n}))}\left(\frac{\mathrm{P}(\mathrm{n}+1)}{\mathrm{P}(\mathrm{n})}-1\right) .
$$

Com a Equação (13) e os valores de h e P da Tabela 1a, calculamos os valores de $\mathrm{r}_{\mathrm{n}}$ cujos resultados encontram-se na Tabela 2. Consideramos, nesse caso, a capacidade de carga $\mathrm{K}=235.000 .000$ habitantes. Estamos supondo que os recursos no Brasil suportam uma população até esse valor de K.

Com esses valores podemos obter uma estimativa da população brasileira em 2020, usando: (i) a média aritmética dos valores de $r_{n}$ e (ii) a média harmônica dos valores de $r_{n}$. Assim,

(i) $\mathrm{r}_{\mathrm{ma}}=\frac{0,4133}{11}=0,0376$.

(ii) $\mathrm{r}_{\mathrm{mh}}=\frac{11}{321,3841}=0,0342$.

Com o modelo Logístico discretizado (12),

$$
\mathrm{P}(12)=\mathrm{P}(11)+\mathrm{h}_{12} \mathrm{r}\left(1-\frac{\mathrm{P}(11)}{\mathrm{K}}\right) \mathrm{P}(11),
$$

obtemos as seguintes projeções da população para 2020:

(i) $\mathrm{P}(12)=204.259 .540$ habitantes com a média aritmética; 
(ii) $\mathrm{P}(12)=203.038 .457$ habitantes com a média harmônica.

Nesse modelo, com o valor de $\mathrm{K}$ usado, o número de habitantes fica abaixo da estimativa do IBGE com ambas as médias. Se mudarmos o valor da capacidade de carga K, por exemplo, $\mathrm{K}=280.000 .000$, e refizermos todos os cálculos acima, obtemos $\mathrm{P}=212.826 .003$ habitantes com a média aritmética e $\mathrm{P}=211.306 .017$ habitantes com a média harmônica. Esses valores aproximamse mais do valor estimado pelo IBGE. Isso mostra também que tal modelo é bastante sensível ao valor de $\mathrm{K}$.

\begin{tabular}{ccc}
\hline $\mathrm{r}_{\mathrm{n}}$ & Modelo Exponencial & Modelo Logístico \\
\hline $\mathrm{r}_{1}$ & 0,0246 & 0,0257 \\
$\mathrm{r}_{2}$ & 0,0217 & 0,0231 \\
$\mathrm{r}_{3}$ & 0,0378 & 0,0409 \\
$\mathrm{r}_{4}$ & 0,0173 & 0,0199 \\
$\mathrm{r}_{5}$ & 0,0260 & 0,0315 \\
$\mathrm{r}_{6}$ & 0,0367 & 0,0471 \\
$\mathrm{r}_{7}$ & 0,0331 & 0,0475 \\
$\mathrm{r}_{8}$ & 0,0282 & 0,0472 \\
$\mathrm{r}_{9}$ & 0,0193 & 0,0399 \\
$\mathrm{r}_{10}$ & 0,0171 & 0,0457 \\
$\mathrm{r}_{11}$ & 0,0125 & 0,0448 \\
\hline
\end{tabular}

Tabela 2: Valores de $\mathrm{r}_{\mathrm{n}}$.

\subsection{Modelo de Gompertz}

Considere a seguinte hipótese:

"A resistência de uma pessoa à morte diminui conforme sua idade aumenta".

Parece uma suposição bem aceitável, não? Pois foi justamente a partir dela que Benjamin Gompertz (1779-1865), um atuário inglês, desenvolveu e publicou, em 1825, seu trabalho intitulado On the Nature of the Function Expressive of the Law of Human Mortality, and on a New Mode of Determining the Value of Life Contingencies [5] sobre crescimento populacional e mortalidade humana. Ele realizou esse estudo ante a necessidade de se determinarem valores mais precisos para os seguros vendidos por sua companhia de seguros.

A expressão matemática resultante desse trabalho ficou conhecida como "Lei de Gompertz", e é dada por

$$
\mathrm{P}(\mathrm{t})=\mathrm{Ke} \mathrm{e}^{\ln \left(\frac{\mathrm{P}_{0}}{\mathrm{~K}}\right) \mathrm{e}^{-\mathrm{rt}}},
$$

onde $\mathrm{P}$ é a população no instante $\mathrm{t}$ e $\mathrm{P}_{0}$ é a população inicial. As constantes $\mathrm{r}$ e $\mathrm{K}$ são positivas vinculadas às particularidades da população em estudo e ao meio onde a população está inserida. A Lei de Gompertz surge como solução do problema de valor inicial

$$
\left\{\begin{array}{l}
\frac{\mathrm{dP}}{\mathrm{dt}}=r \mathrm{P} \ln \left(\frac{\mathrm{K}}{\mathrm{P}}\right) \\
\mathrm{P}(0)=\mathrm{P}_{0}
\end{array}\right.
$$


que, na verdade, trata-se de certo modo de um refinamento do modelo de Malthus (Exponencial). Ver isso é uma tarefa simples, pois através da mudança $\omega=\ln \left(\frac{\mathrm{K}}{\mathrm{P}}\right)$ chegamos facilmente a

$$
\mathrm{P}^{\prime}=-\mathrm{P} \omega^{\prime}
$$

e substituindo na EDO em (16) conclui-se que

$$
\omega^{\prime}=-\mathrm{r} \omega
$$

que é o modelo Exponencial.

Cabe comentar que Gompertz inicialmente "publicou" seu artigo como uma carta pessoal para Francis Baily e, ao construir sua lei, considerou apenas a mortalidade humana advinda do fator idade e, por essa razão, é um modelo chamado de dependente da idade (age-dependent model).

Posteriormente, Willian Matthew Makeham (1826-1891) incluiu no modelo de Gompertz fator não dependente da idade, incluindo acidentes por exemplo. Daí surgiu então um dos mais completos estudos sobre a mortalidade humana que acabou conhecido como a Lei de Gompertz-Makeham, que descreve com muita acurácia a mortalidade humana entre as idades de 20 a 80 anos.

O trabalho de Makeham que completou Gompertz foi intitulado On the Law of Mortality and the Construction of Annuity Tables [6].

Podemos visualizar o comportamento da família das soluções da equação diferencial (16) na Figura 3.

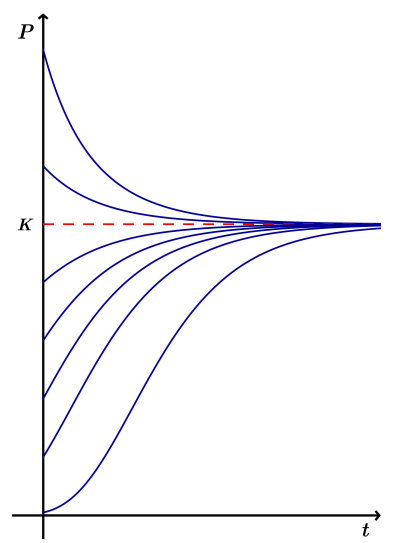

Figura 3: Esboço da Solução Geral da equação de Gompertz.

\subsubsection{Discretização da Equação de Gompertz}

Aplicando o modelo de Euler (2) na equação diferencial de Gompertz (16), obtemos

$$
\mathrm{P}(\mathrm{n}+1)=\mathrm{P}(\mathrm{n})+\mathrm{h}_{\mathrm{n}} \mathrm{r}_{\mathrm{n}} \mathrm{P}(\mathrm{n}) \ln \left(\frac{\mathrm{K}}{\mathrm{P}(\mathrm{n})}\right),
$$

e com manipulações algébricas elementares podemos escrever

$$
r_{n}=\frac{P(n+1)-P(n)}{h_{n} P(n) \ln \left(\frac{K}{P(n)}\right)} .
$$


Assim, com a Equação (18) e os valores de h e P da Tabela 1a, calculamos os valores de $\mathrm{r}_{\mathrm{n}}$ cujos resultados encontram-se na Tabela 3. Note que, aqui e nos outros modelos anteriores, consideramos a capacidade de carga como sendo $K=235.000 .000$. Trata-se de uma valor de difícil determinação, e adotamos tal valor pois as estimativas da população brasileira fornecidas oficialmente pelo IBGE [7] que estão na Tabela 1b tendem a ter um teto não superior a 235.000.000.

Como anteriormente, utilizamos média aritmética e média harmônica para chegar a dois valores de r, e, então, estimarmos a população brasileira para 2020 e adiante. Assim, com o modelo de Gompertz discretizado (17),

$$
\mathrm{P}(12)=\mathrm{P}(11)+\mathrm{h}_{12} \mathrm{rP}(11) \ln \left(\frac{\mathrm{K}}{\mathrm{P}(11)}\right),
$$

obtemos as seguintes projeções da população para 2020:

(i) $\mathrm{P}(12)=202.133 .586$ habitantes com a média aritmética;

(ii) $\mathrm{P}(12)=199.321 .631$ habitantes com a média harmônica.

As estimativa do IBGE para a população brasileira em 2020 é de 211.755.692 indivíduos. Note que, assim como o caso Logístico, Gompertz estimou um crescimento mais tímido do que as previsões oficiais.

\begin{tabular}{cc}
\hline $\mathrm{r}_{\mathrm{n}}$ & Modelo de Gompertz \\
\hline 1 & 0,0075 \\
2 & 0,0075 \\
3 & 0,0140 \\
4 & 0,0081 \\
5 & 0,0141 \\
6 & 0,0228 \\
7 & 0,0255 \\
8 & 0,0279 \\
9 & 0,0253 \\
10 & 0,0300 \\
11 & 0,0292 \\
\hline
\end{tabular}

Tabela 3: Valores de $r_{n}$.

\subsection{Visão geral dos dados}

Esta subseção visa apenas compilar os dados dos três modelos em um único local para facilidade de análise.

Começamos com as estimativas populacionais. Note que, semelhantemente ao cálculo feito para estimarmos a população em 2020, fizemos os respectivos cálculos para 2030, 2040, 2050 e 2060. Utilizamos, no entanto, em cada modelo, os mesmos valores de r que foram utilizados para calcular a estimativa 2020 . 


\begin{tabular}{cc|cc|cc||cc}
\hline & & \multicolumn{2}{c|}{ Modelo Exponencial } & \multicolumn{2}{c||}{ Modelo Logístico } & \multicolumn{2}{c}{ Modelo de Gompertz } \\
\hline Ano & IBGE & ma & mh & ma & mh & ma & mh \\
\hline 2020 & 211.755 .692 & 238.253 .993 & 233.294 .342 & 204.259 .540 & 203.038 .457 & 199.426 .046 & 196.887 .442 \\
2030 & 224.868 .462 & 297.579 .237 & 291.384 .633 & 214.305 .992 & 212.482 .637 & 206.558 .828 & 202.256 .268 \\
2040 & 231.919 .922 & 371.676 .467 & 356.363 .406 & 221.401 .752 & 219.445 .682 & 212.365 .023 & 206.932 .979 \\
2050 & 232.933 .276 & 464.223 .907 & 435.832 .445 & 226.218 .834 & 224.413 .164 & 217.051 .638 & 210.988 .878 \\
2060 & 228.286 .347 & 579.815 .659 & 533.023 .080 & 229.397 .178 & 227.870 .748 & 220.809 .280 & 214.493 .174 \\
\hline
\end{tabular}

Tabela 4: Estimativas da população do IBGE e modelos de 2020 a 2060, com média aritmética (ma) e com média harmônica (mh).

As próximas duas figuras são gráficos de pontos traduzindo os valores constantes na Tabela 4 . Separamos em dois gráficos pois o modelo Exponencial é muito discrepante em relação aos outros conforme o tempo passa, e a visualização ficaria prejudicada devido ao tamanho.

Inicialmente, na Figura 4, comparamos os dados oficiais com os resultados do Modelo Exponencial.

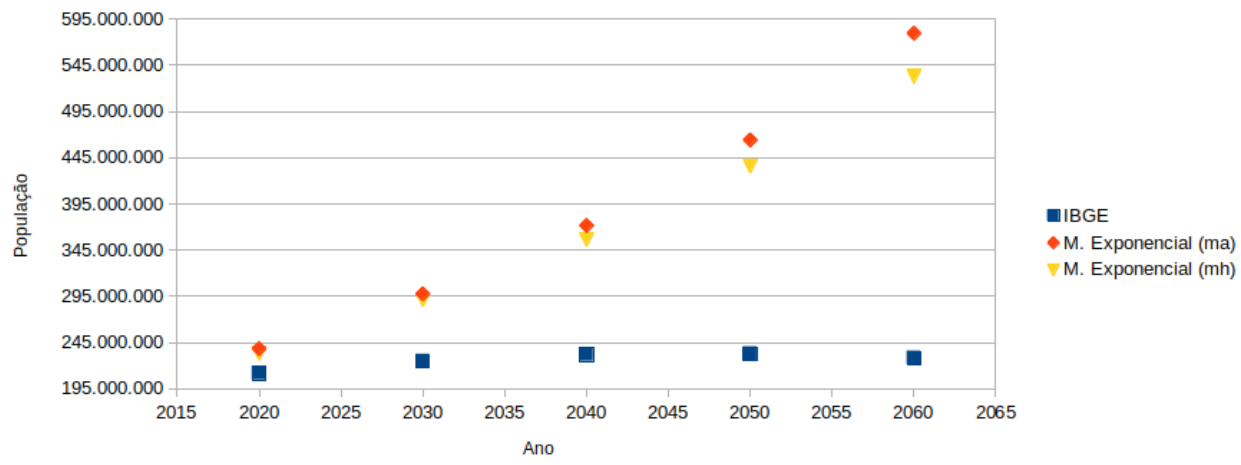

Figura 4: Estimativas com dados do IBGE e modelo Exponencial.

Para completar os gráficos de pontos, na Figura 5 comparamos os dados oficiais com os resultados dos modelos Logístico e de Gompertz.

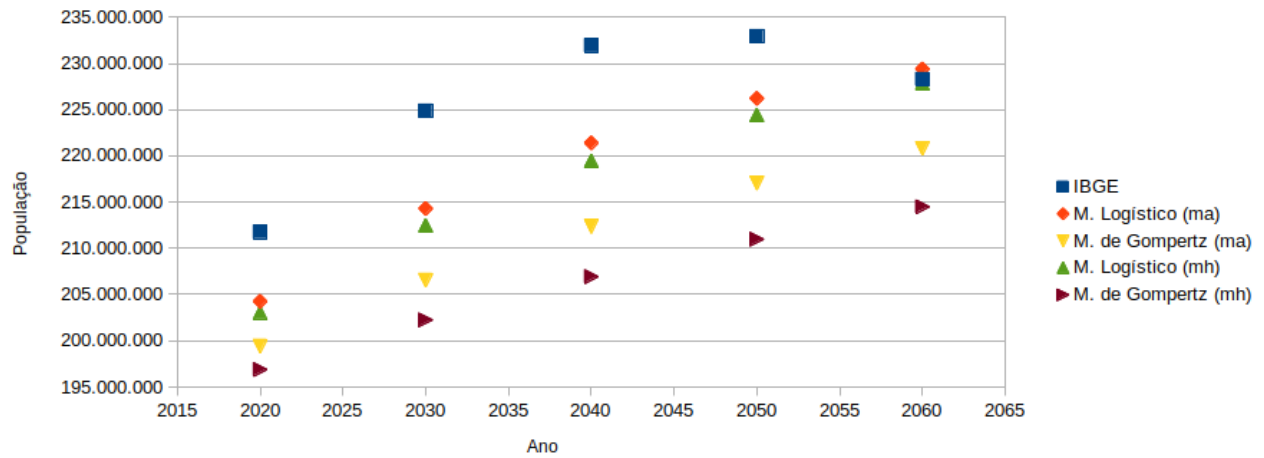

Figura 5: Estimativas com dados do IBGE e modelos Logístico e de Gompertz.

E, por fim, adicionamos a Tabela 5 com comparativos percentuais de cada modelo relativamente às previsões oficiais. Note que os sinais negativos são devidos ao fato de que tanto o modelo Logístico quanto o de Gompertz, em geral, subestimam a população, enquanto que o Exponencial superestima. 


\begin{tabular}{ccccccc}
\hline & \multicolumn{2}{|c|}{ Modelo exponencial } & \multicolumn{2}{c|}{ Modelo logístico } & \multicolumn{2}{c}{ Modelo de Gompertz } \\
\hline Ano & ma (\%) & mh (\%) & ma (\%) & mh (\%) & ma (\%) & mh (\%) \\
\hline 2020 & 12,5 & 10,2 & $-3,5$ & $-4,1$ & $-5,8$ & $-7,0$ \\
2030 & 32,3 & 29,6 & $-3,4$ & $-5,5$ & $-8,1$ & $-10,1$ \\
2040 & 60,3 & 53,7 & $-4,5$ & $-5,4$ & $-8,4$ & $-10,8$ \\
2050 & 94,3 & 87,5 & $-2,9$ & $-3,7$ & $-6,8$ & $-9,4$ \\
2060 & 154,0 & 133,5 & 0,5 & $-0,2$ & $-3,3$ & $-6,0$ \\
\hline
\end{tabular}

Tabela 5: Diferença percentual em relação aos valores do IBGE, com média aritmética (ma) e com média harmônica $(\mathrm{mh})$.

\section{Proposta de atividade e considerações}

Este conteúdo como apresentado oportuniza inúmeras possibilidades de atividades com turmas de Ensino Médio. Uma delas, que nos parece bastante óbvia e comum e que por isso deve se encaixar em praticamente toda turma, é a de que o professor separe seus alunos em grupos e distribua os modelos entre esses grupos. Para esclarecer e facilitar, as atividades podem ser divididas em etapas.

- Inicialmente peça um trabalho superficial sobre censo onde os grupos deverão buscar e reunir num único trabalho geral informações sobre cada etapa do censo brasileiro.

- Numa segunda etapa o professor deverá indicar a cada grupo o modo de operar com a respectiva equação, passando os detalhes matemáticos no bom e velho esquema de giz e saliva, e instruindo-os como utilizar a calculadora ou o computador. Note que essa é uma parte trabalhosa do processo, uma vez que cabe ao professor explicar o respectivo modelo para cada grupo individualmente. Antes disso, sugerimos que haja uma breve explanação sobre a origem dessas fórmulas. Neste momento o professor não precisa (e nem deve) explanar sobre equações diferenciais mas apenas citar ser esse um conteúdo que se aprende lá nas faculdades de ciências exatas e que ali os modelos já foram discretizados. Trata-se de uma boa oportunidade para citar conteúdos estatísticos associados às médias e percentuais, probabilidade, gráficos de funções e ir além das famosas progressões aritméticas e geométricas e introduzir outros tipos de sequências numéricas. Isso só pra falar das associações óbvias.

- A terceira etapa seria a compilação desses dados em gráficos e tabelas e, embora simples, aqui pode haver alguma dificuldade se a escola não prover as ferramentas computacionais necessárias. Essa etapa é autoexplicativa e requer apenas acompanhamento de perto por parte do professor, ao contrário da etapa anterior, que requer acompanhamento muito de perto pelo professor.

- A quarta etapa poderia ser a compilação dos resultados em cartazes e a exposição dos dados em algum ambiente com acesso dos demais alunos da escola. Se os resultados forem apresentados na iminência da divulgação dos dados oficiais, haverá, inclusive, um fator motivacional agregado nessa tarefa o que sabemos facilitar muito o trabalho do educador.

- Na quinta, e opcional, etapa o professor poderia indagar a turma sobre a maneira oficial adotada pelo IBGE para contar e estimar a população brasileira. Trata-se de uma etapa difícil, que irá requerer um bom estudo adicional por parte do professor para entender e, de certo modo, dominar o Método das Componentes Demográficas (MCD) que pode ser acessado em [8]. 
Sabemos, no entanto que, em geral, professores de educação básica dispõem de pouco tempo para si e que essa etapa é de difícil implementação no que tange tempo, conteúdo programático e interesse dos alunos. Por isso a colocamos como opcional.

Claro, vale lembrar que tudo isso são apenas sugestões de como proceder com o conteúdo aqui apresentado. Sem sombra de dúvidas, o professor é incentivado a implementar esse material alterando totalmente o plano de ação em sala de aula.

Dando sequência às nossas considerações acerca desse tema gostaríamos de falar um pouco sobre todo o conteúdo matemático que se pode chamar à atenção e destacar nesta atividade. Note que inicialmente o professor já começa citando equações diferenciais. Claro que não vai falar em detalhes, pois nem caberia, mas o simples citar é de fundamental importância, uma vez que é difícil o interesse por algo do qual sequer ouviu falar.

Adicionalmente, note que é uma excelente maneira de se contextualizar diversos conteúdos utilizados e tratados no Ensino Médio ou anterior, como sequências numéricas, funções, porcentagem e médias.

Outro fator de importância muito atual e que é tratado de forma no mínimo insuficiente na educação brasileira são as aproximações numéricas.

Além dos conteúdos matemáticos citados, note também que uma outra alternativa de discussão apresenta-se diante do professor e sua turma: O que é ciência? O que são modelos científicos?

Diante do histórico de desenvolvimento dos modelos populacionais, os resultados aproximados e a diferença do método adotado hoje e suas previsões, pode-se levantar a pergunta sobre a evolução dos modelos e concluir que ciência não é atividade pronta e incontestável mas sim o contrário; indagar, questionar e duvidar são etapas essenciais na confecção do conhecimento científico que realmente levam à evolução.

É de nossa firme opinião que não se deve ensinar ciência, em especial matemática, como um conjunto imutável de conhecimento com fórmulas prontas, e criar o receio de contestar, nem tampouco associar a essa disciplina a noção de que a origem do que se trata é mentalmente intangível. Infelizmente isso é prática comum em nossas salas de aula.

Sendo assim, para cada um desses assuntos associados, o professor precisa traçar uma estratégia de abordagem, considerando modo e profundidade no tratar. E é justamente através dessa última frase que entramos em mais um tópico ao qual gostáriamos de apresentar algumas ideias: a formação básica de Licenciados em Matemática.

Segundo a BNCC [1], para o ensino médio, o educador deve atuar para desenvolver habilidades que possam favorecer a interpretação e compreensão da realidade pelos estudantes, através da aplicação de conceitos de diferentes campos da matemática na interpretação de situações em diversos contextos, sejam atividades cotidianas, sejam fatos das ciências da natureza e humanas, das questões socioeconômicas ou tecnológicas de modo a contribuir para uma formação geral sólida.

A pergunta que fazemos é: Como pode um professor de matemática desenvolver (e aprimorar) uma atividade como essa proposta neste artigo sem jamais ter tido uma boa fundamentação em Análise Matemática e Equações Diferenciais? A resposta é bem simples: não pode!

Sendo assim, esperamos também chamar a atenção sobre o fato de que não se ensina o não sabido, e que, portanto, é fundamental que universidades proporcionem uma formação técnica sólida para seus licenciandos (no caso, em matemática). É preciso que licenciados em matemática tenham 
conhecimentos em matemática avançada: Análise, Geometria Diferencial, Topologia, Álgebra e Lógica. E que saibam para que tais áreas servem e onde se aplicam. Nesse sentido, é oportuno também incentivar, e dar o devido tempo, para que professores aprimorem seus conhecimentos matemáticos e cubram eventuais lacunas de formação. Acreditamos que manter um processo permanente e constante de aperfeiçoamento de conhecimentos necessários às atividades dos educadores proporciona um ensino de qualidade aos estudantes.

Assim, esperamos que este artigo desperte e contribua com a real necessidade de uma Licenciatura em Matemática forte no que se refere à formação de um educador competente, dotado de espírito crítico e criativo, capaz de compreender e relacionar a matemática com outros segmentos, colaborando de fato para o desenvolvimento humano da região na qual está inserido. Que esse professor atue com uma visão abrangente do papel da matemática como campo de conhecimento, elabore propostas alternativas para a sala de aula, e que se convença de que os conceitos de matemática superior, abordados neste artigo, permitem-lhe aprofundar os conhecimentos e o ensinar dos programas do ensino médio para que possa transmitir uma visão da importância dos tópicos que esteja lecionando. Esperamos que este trabalho contribua para mostrar aos educadores e estudantes como utilizar estratégias, conceitos e procedimentos matemáticos para interpretar uma situação-problema, construir modelos e determinar soluções analisando a coerência dos resultados obtidos de modo a construir uma argumentação consistente e, no fim, realmente aprimorar o educar.

\subsection{E doravante?}

O trabalho não acaba por aqui. Sugerimos que você, professor, adote outros modelos populacionais com sua turma, faça a devida pesquisa quanto a origem, autor, por qual razão foi proposto e, claro, discretize-o.

Um modelo que segue naturalmente os propostos no texto e que pode ser adotado em adição ou em substituição a eles é o de crescimento Logístico com Limiar,

$$
\left\{\begin{array}{l}
\frac{\mathrm{dP}}{\mathrm{dt}}=-\mathrm{r}\left(1-\frac{\mathrm{P}}{\mathrm{T}}\right)\left(1-\frac{\mathrm{P}}{\mathrm{K}}\right) \mathrm{P} \\
\mathrm{P}(0)=\mathrm{P}_{0},
\end{array}\right.
$$

onde $\mathrm{r}>0$ e $0<\mathrm{T}<\mathrm{K}$, que introduz o limiar $\mathrm{T}$ e faz com que $\frac{\mathrm{dP}}{\mathrm{dt}}$ fique negativo quando $\mathrm{P}$ fique grande, o que irá proporcionar decréscimo populacional, como observado na Tabela 1b oficial do IBGE.

Para um tratamento mais completo e sugestão de novos modelos para incluir na atividade recomendamos o livro [3].

\section{Referências}

[1] Base Nacional Comum (BNCC), http://basenacionalcomum.mec.gov.br/.

[2] Boyce, W.E., DiPrima, R.C., Equações Diferenciais Elementares e Problemas de Valores de Contorno. LTC Editora. 10a Edição. 2015.

[3] Frauenthal, J.C., Introduction to Population Modeling. Boston: Birkhauser, 1980.

[4] Goldoni, E.K.S., Matemática aplicada ao estudo da área ocupada pelo crescimento de microorganismos como ferramenta para o ensino da função exponencial, PMO v.7, nº, 2019, https://doi.org/10.21711/2319023x2019/pmo712. 
[5] Gompertz, B., On the Nature of the Function Expressive of the Law of HumanMortality, and on a New Mode of Determining the Value of Life Contingencies, Philosophical Transactions of the Royal Society of London, v. 115 (1825), pp.513-583.

[6] Gompertz, B., Makeham, W. M., On the Law of Mortality and the Construction of Annuity Tables, Cambridge University Press.

[7] IBGE, https://www.ibge.gov.br.

[8] IBGE - MCD, https://www.ibge.gov.br/apps/populacao/projecao/notatecnica.html.

[9] Malthus, T.R., An Essay of the Principle of Population, Printed for J. Johnson in St. Paul's Church-Yard, V1, Fourth Edition, 1807.

[10] Nagle, R.K., Saff, E.B., Snides, A.D., Equações Diferenciais, Editora Pearson Education do Brasil Ltda, 8a Edição, 69-70, 2012.

[11] Verhulst, P.F., Notice sur la loi que la population suit dans son accroissement. Correspondances Mathematiques et Physiques, 10, 113-121, 1838.

José Rafael Santos Furlanetto

Universidade do Estado de Santa Catarina $<$ jose.furlanetto@udesc.br>

Ligia Liani Barz

Universidade do Estado de Santa Catarina $<$ ligia.barz@udesc.br>

Recebido: $20 / 04 / 2020$

Publicado: $19 / 06 / 2020$ 